\title{
The levels of SYT13 and CEA mRNAs in peritoneal lavages predict the peritoneal recurrence of gastric cancer
}

\author{
Koki Nakanishi ${ }^{1} \cdot$ Mitsuro Kanda $^{1}$ (1) -Shinichi Umeda ${ }^{1} \cdot$ Chie Tanaka $^{1} \cdot$ Daisuke Kobayashi $^{1} \cdot$ Masamichi Hayashi $^{1}$. \\ Suguru Yamada ${ }^{1} \cdot$ Yasuhiro Kodera ${ }^{1}$
}

Received: 21 January 2019 / Accepted: 25 April 2019 / Published online: 4 May 2019

(c) The International Gastric Cancer Association and The Japanese Gastric Cancer Association 2019

\begin{abstract}
Background Although peritoneal lavage cytology often serves as a sensitive method to detect free cancer cells in the abdominal cavity, some patients experience peritoneal recurrence despite negative cytology. The aim of this study was to evaluate mRNAs in peritoneal lavage fluid as potential markers for predicting the peritoneal recurrence of gastric cancer (GC).

Methods Peritoneal lavage fluid samples were obtained during surgery conducted on 187 patients with GC and from 30 patients with non-malignant disease (controls). The mRNA levels of nine candidate markers were quantified, and analysis of a receiver-operating characteristic curve compared their accuracies. The cutoff was defined as the highest value of the controls. Results Synaptotagmin XIII (SYT13) and carcinoembryonic antigen (CEA) mRNA levels were analyzed further. SYT13 levels were significantly associated with shorter peritoneal recurrence-free survival (PRFS) and overall survival. Among patients with negative peritoneal lavage cytology, those positive for either SYT13 or CEA mRNA experienced significantly shorter peritoneal recurrence-free survival compared with those with negative fluid (hazards ratio [HR] 4.21, $P=0.0114$; HR 3.53; $P=0.0426$, respectively). Univariate analysis revealed that $S Y T 13$ and $C E A$ mRNA levels were significant predictors of peritoneal recurrence. Positive levels of both SYT13 and CEA mRNA demonstrated the highest HR for peritoneal recurrence (HR 12.27, $P=0.0064)$. Multivariable analysis revealed that $S Y T 13$ positivity was a significant independent prognostic factor (HR 3.69; 95\% confidence interval, 1.18-12.74; $P=0.0246$ ).

Conclusions Combined measurement of SYT13 and CEA mRNA levels in peritoneal lavage fluid could serve as a promising approach to predict peritoneal recurrence of GC.
\end{abstract}

Keywords Gastric cancer $\cdot$ Peritoneal metastasis $\cdot$ Biomarker $\cdot$ Synaptotagmin XIII $\cdot$ Carcinoembryonic antigen

\section{Introduction}

Gastric cancer is the fifth most common malignancy worldwide [1]. Peritoneal dissemination is the second most common cause of recurrence after liver metastases and is associated with poor survival [2,3]. Peritoneal lavage cytology is considered as a practical method for detecting free cancer cells in the peritoneal cavity and for predicting

Electronic supplementary material The online version of this article (https://doi.org/10.1007/s10120-019-00967-3) contains supplementary material, which is available to authorized users.

Mitsuro Kanda

m-kanda@med.nagoya-u.ac.jp

1 Department of Gastroenterological Surgery (Surgery

II), Nagoya University Graduate School of Medicine, 65

Tsurumai-cho, Showa-ku, Nagoya 466-8550, Japan peritoneal metastasis $[4,5]$, but unfortunately suffers from limitations in terms of sensitivity [5, 6]. Genetic detection using reverse transcriptase-polymerase chain reaction (RTPCR) has been attempted to improve diagnostic accuracy [7-9]. This information may contribute to the identification of patients at high risk of peritoneal recurrence and could identify candidate patients for aggressive perioperative treatment to combat intraperitoneal disease (e.g., intraperitoneal chemotherapy and cytoreductive surgery plus hyperthermic intraperitoneal chemotherapy).

Carcinoembryonic antigen (CEA), cytokeratin 20 (CK20), cytokeratin 19 (CK19), melanoma-associated gene (MAGE), and matrix metalloproteinase-7 (MMP7) are target genes that serve as sensitive and specific markers for gastric cancer [8-11]. Multiple molecular markers and microarrays are also used for this purpose [10-15]. According to a systematic review and meta-analyses, 
well-validated $C E A$ assays are superior to others for analyzing gastric cancers [8, 9]. However, there is ample room for improvement in diagnostic accuracy, given that the conventional assays have not yet been translated to the clinic [16].

We recently identified several novel molecules, each of which served as independent risk factors for peritoneal recurrence in gastric cancer [17-21]. We hypothesized that the mRNA levels of these molecules in peritoneal lavage fluid could predict peritoneal recurrence and prognosis. To identify biomarkers that could replace or augment $C E A$, we conducted quantitative real-time reverse transcriptionPCR (qRT-PCR) analyses of nine candidate markers and evaluated their clinical relevance.

\section{Methods}

\section{Patients and sample collection}

Peritoneal lavage fluid samples were collected from 187 patients with gastric adenocarcinoma who underwent open gastrectomy at the Department of Gastroenterological Surgery, Nagoya University Hospital, Japan, between August 2002 and March 2018. To ensure the consistent collection of the samples and the quality of the gastrectomies, we excluded patients who underwent laparoscopic surgery as well as those with macroscopic peritoneal metastasis, and those who received preoperative treatment. The disease stages of patients were determined according to the 8th edition of TNM staging system of the Union for International Cancer Control [22]. Postoperative chemotherapy was administered at the discretion of the surgeons. Since 2006, adjuvant S-1 (an oral fluoropyrimidine derivative) monotherapy has been recommended to all patients with stage II/III gastric cancer, unless contraindicated by the patient's condition. For patients in stage IV and those who experienced recurrence, systematic chemotherapy according to the guidelines at that time (mainly, $\mathrm{S}-1$ plus cisplatin or S-1 monotherapy) was administered at the physician's discretion. Among them, the expression levels of SYT13 mRNA in corresponding primary gastric cancer tissues were obtained from a previous study [17] and were used for correlation analysis with the expression levels in the lavage fluid samples.

To serve as controls, peritoneal lavage fluids were also collected from 30 patients who underwent laparotomy for non-malignant diseases (e.g., gallstones and abdominal incisional hernias) between December 2014 and June 2016. We used CT and endoscopy to determine that there were no detectable malignancies.

\section{Ethics}

All procedures were conducted in accordance with the ethical standards of the responsible committee on human experimentation (institutional and national) and with the Declaration of Helsinki (1964) and later versions. Informed consent to be included in the study, or equivalent, was obtained from all patients as required by the Institutional Review Board of Nagoya University, Japan.

\section{Sample processing}

Immediately after laparotomy, $200 \mathrm{ml}$ of saline was introduced into the recto-uterine pouch (Douglas pouch) and aspirated soon after gentle stirring. Half of each fluid sample was analyzed by routine cytopathology (conventional Papanicolaou and Giemsa staining), while the other half was used to measure mRNA levels. For patients with benign disease, $100 \mathrm{ml}$ of saline was introduced immediately after laparotomy into the recto-uterine pouch, which was aspirated soon after gentle stirring and used to measure mRNA levels. Samples were centrifuged at $3000 \times g$ for $10 \mathrm{~min}$ to collect intact cells, which were rinsed with phosphate-buffered saline, dissolved in ISOGEN-LS RNA extraction buffer (Nippon Gene, Tokyo, Japan), and stored immediately in liquid nitrogen at $-80{ }^{\circ} \mathrm{C}$.

\section{mRNA extraction and RT-PCR analysis}

Total RNA was extracted from each frozen sample using an RNeasy Mini Kit (Qiagen, Hilden, Germany). A quality check of RNA samples was conducted before generating cDNA. The ratios of absorbance at 260 to $280 \mathrm{~nm}$ of RNA ranged from 1.8 to 2.0. Total RNA (10 $\mu \mathrm{g}$ per sample) was isolated and used as template for cDNA synthesis. mRNA levels were determined using a qRT-PCR assay, and real-time detection of SYBR Green fluorescence intensity was performed using an ABI StepOnePlus RealTime PCR System (Applied Biosystems, Foster City, CA, USA). We calculated the mRNA levels of synaptotagmin VIII (SYT8), synaptotagmin XIII (SYT13), sushi domain containing 2 (SUSD2), melanoma-associated gene family member D4 (MAGED4), dihydropyrimidinase-like 3 (DPYSL3), anosmin-1 (ANOS1), CK19, CK20, and CEA. The level of glyceraldehyde-3-phosphatedehydrogenase $(G A P D H)$ mRNA was quantified in each sample and used to normalize the data. Nucleotide sequences for primers and probes are listed in Supplemental Table 1. The PCRs were performed as follows: initial denaturation at $95{ }^{\circ} \mathrm{C}$ for $10 \mathrm{~min}, 40$ cycles at $95{ }^{\circ} \mathrm{C}$ for $10 \mathrm{~s}$, and at $60{ }^{\circ} \mathrm{C}$ for $30 \mathrm{~s}$. All samples were tested in triplicate, and samples 
Table 1 Analysis of the levels of SYT13 and CEA mRNAs in the peritoneal lavage fluids

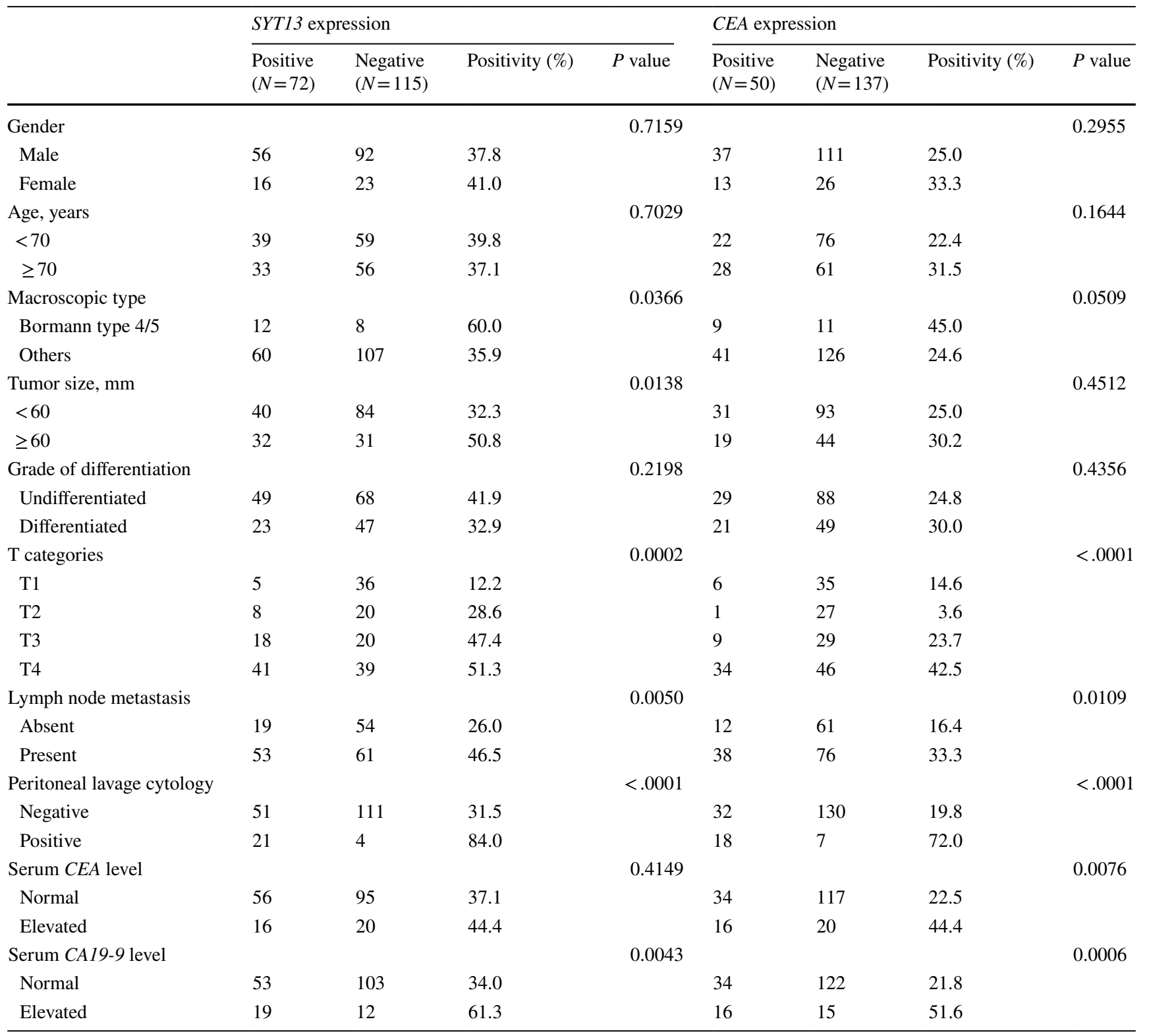

SYT13: Synaptotagmin XIII, CEA: Carcinoembryonic antigen, CA19-9: Cancer antigen 19-9

without template were included in each PCR plate as negative controls.

\section{Follow-up}

Patients who underwent curative resection were followed according to the Japanese guidelines version 4 [23]. Physical examinations and laboratory tests were performed every 3 months for the first 3 years and every 6 months for 5 years or until death after surgery. Enhanced CT (chest and abdominal cavity) was conducted every 6 months for 5 years. Patients receiving chemotherapy underwent hematologic tests and assessments of clinical symptoms every cycle. Patients were diagnosed with peritoneal recurrence when peritoneal nodules were detected by imaging studies, or when peritoneal metastasis including positive cytology was detected using staging laparoscopy.

\section{Statistical analysis}

Receiver-operating characteristic (ROC) curves were generated to compare the accuracies of candidate markers. Correlations between the PCR data and clinicopathological variables were analyzed using $\chi^{2}$ and Fisher's exact tests. Overall survival (OS) and peritoneal recurrence-free survival (PRFS) were calculated using the Kaplan-Meier 
method, and differences in survival curves were analyzed using the log-rank test. The Cox proportional hazards model was used for multivariate analysis after relevant prognostic variables were identified using univariate analysis. When we analyzed PRFS, the data for patients who died from other recurrences were censored. Data were analyzed using JMP ver13 software (JMP, SAS Institute, Cary, North Carolina, USA). $P<0.05$ indicates significant differences.

\section{Results}

\section{Patients' demographics}

The study included 148 men and 39 women with a combined median age of 69 years (range 20-90 years). Patients underwent total gastrectomy $(n=72,38.5 \%)$, distal gastrectomy ( $n=98,52.4 \%)$, or limited gastrectomy $(n=17,9.1 \%)$, which is indicated for early gastric cancer. Patients were staged as follows: $n=55$, stage I (IA: 36 , IB: 19); $n=39$, stage II (IIA: 19, IIB: 20); $n=63$, stage III (IIIA: 18, IIIB: 24, IIIC: 21 ); and $n=30$, stage IV. Among stage IV patients, 25 underwent positive peritoneal lavage cytology (CY1; surgical R1 resection), two underwent gastrectomy with partial hepatectomy (surgical R0 resection) for synchronous liver metastases, and three underwent extended lymphadenectomy beyond D2 (surgical R0 resection). Postoperative chemotherapy was administered to 79 patients (42.2\%). The median follow-up after surgery was 38.1 months or until death. Patients' demographics are summarized in Supplemental Table 2.

\section{Selection of molecular markers}

Receiver operating characteristic (ROC) curves were generated to compare the predictive values of the mRNA/GAPDH ratios of nine candidate markers. We determined the area under the curve (AUC) values for positive peritoneal lavage cytology, peritoneal recurrence, or both. The AUC values were as follows: $0.775, C E A ; 0.771$, SYT13; 0.581, SUSD2; 0.568, DPYSL3; 0.562, MAGED4; 0.551, CK19; 0.542, CK20; 0.533, ANOS1, and 0.508, SYT8. Thus, we selected $S Y T 13$ and $C E A$ for further analyses.

\section{SYT13 and CEA mRNA levels in peritoneal lavage fluid}

The ratios of $S Y T 13 / G A P D H$ and $C E A / G A P D H$ mRNAs in peritoneal lavage fluid samples harvested from patients with gastric cancer ranged from 0 to $2.292 \times 10^{-2}$ and 0 to $1.660 \times 10^{-2}$, respectively. Those obtained from patients with non-malignant diseases ranged from 0 to $1.764 \times 10^{-6}$ and from 0 to $3.220 \times 10^{-5}$, respectively (Fig. 1a). The cutoff was defined as the highest value among the control samples, and levels that exceeded the cutoff were defined as positive. The Spearman's rank correlation coefficient between the levels of SYT13 and CEA mRNAs in the peritoneal lavage fluid samples of patients with gastric cancer was 0.3443 (Fig. 1b).

Correlations between peritoneal lavage fluid samples positive for SYT13 and CEA and clinicopathological variables are summarized in Table 1 . Among 187 patients, 72 (38.5\%) were $S Y T 13$ positive and $66(36.5 \%)$ were $C E A$ positive. $S Y T 13$ positivity was significantly correlated with $4 / 5$ macroscopic tumor type, tumors $\geq 60 \mathrm{~mm}$, pT4, lymph node metastasis, positive peritoneal lavage cytology, and elevation of serum CA19-9 levels. SYT13 positivity was not significantly associated with age, sex, tumor differentiation, or serum $C E A$ levels. $C E A$ positivity was significantly correlated with $\mathrm{pT} 4$, lymph node metastasis, positive peritoneal lavage cytology, and elevation of serum CEA and CA19-9

Table 2 Univariate and multivariable analyses of peritoneal recurrence-free survival of patients without concomitant peritoneal metastasis and negative for lavage cytology

\begin{tabular}{|c|c|c|c|c|c|c|}
\hline \multirow[t]{2}{*}{ Variables } & \multicolumn{3}{|l|}{ Univariate } & \multicolumn{3}{|l|}{ Multivariable } \\
\hline & Hazard ratio & $95 \% \mathrm{CI}$ & $P$ value & Hazard ratio & $95 \% \mathrm{CI}$ & $P$ value \\
\hline Gender (male) & 1.12 & $0.25-3.65$ & 0.8695 & & & \\
\hline Tumor size ( $\geq 60 \mathrm{~mm})$ & 2.83 & $0.94-8.82$ & 0.0064 & 1.51 & $0.49-4.83$ & 0.4676 \\
\hline Tumor depth (pT4) & 11.05 & $2.96-71.48$ & 0.0001 & 4.94 & $1.01-37.00$ & 0.0487 \\
\hline Lymph node metastasis (pN2, 3) & 10.78 & $2.88-69.89$ & 0.0002 & 3.40 & $0.69-25.66$ & 0.1381 \\
\hline Macroscopic type (type 4 or 5 ) & 4.32 & $0.97-14.18$ & 0.0547 & & & \\
\hline Invasive growth & 3.27 & $1.08-10.18$ & 0.0358 & 1.32 & $0.37-4.71$ & 0.6626 \\
\hline Tumor differentiation (undifferentiated) & 8.13 & $1.60-148.06$ & 0.0074 & 4.63 & $0.75-90.77$ & 0.1071 \\
\hline Postoperative chemotherapy & 0.63 & $0.19-1.89$ & 0.4090 & & & \\
\hline SYT13 positive & 4.21 & $1.39-14.11$ & 0.0114 & 3.69 & $1.18-12.74$ & 0.0246 \\
\hline$C E A$ positive & 3.53 & $1.05-10.85$ & 0.0426 & 2.30 & $0.62-8.21$ & 0.2026 \\
\hline
\end{tabular}

SYT13 synaptotagmin XIII, CEA carcinoembryonic antigen, $C I$ confidence interval 
Fig. 1 SYT13 and CEA mRNA levels in peritoneal lavage fluid. a Levels of synaptotagmin XIII (SYT13) and carcinoembryonic antigen (CEA) mRNA in peritoneal lavage fluid. $\mathbf{b}$ Scatterplot of SYT13 and CEA mRNA levels
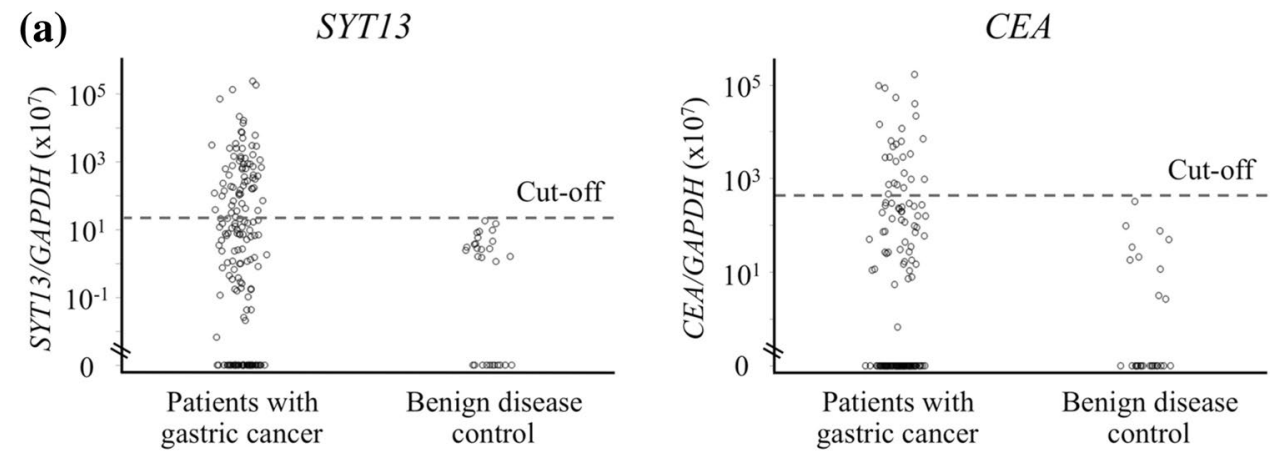

(b)

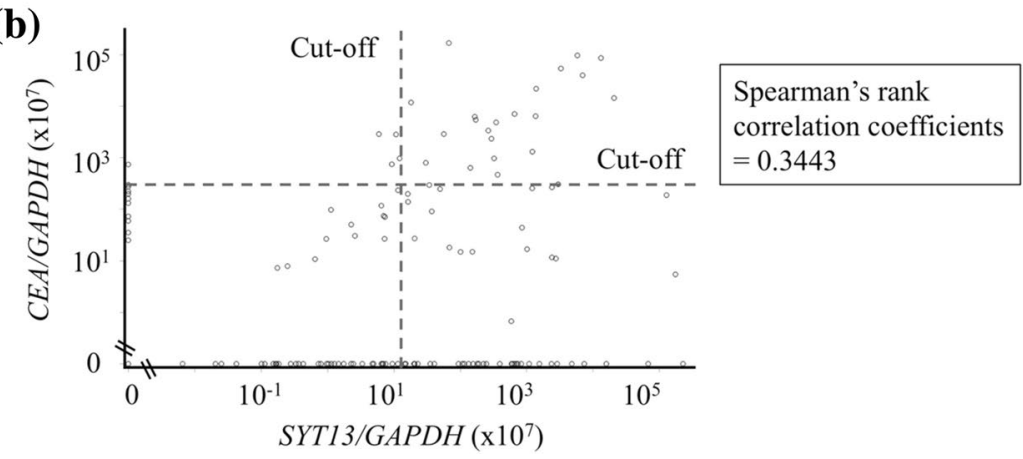

levels. $C E A$ positivity was not significantly associated with age, sex, macroscopic type, or tumor size.

\section{Correlation analysis of SYT13 mRNA levels in peritoneal lavage fluid and primary gastric cancer tissue}

Among the patients included in this study, SYT13 mRNA expression data obtained in corresponding primary gastric cancer tissues of 58 cases were obtained from a previous study. The expression levels of SYT13 mRNA in primary gastric cancer tissue and peritoneal lavage fluid ranged from $1.689 \times 10^{-3}$ to $4.946 \times 10^{-1}$ and 0 to $1.761 \times 10^{-2}$, respectively. The Spearman's rank correlation coefficient between the levels of SYT13 in peritoneal lavage fluid and primary gastric cancer tissue was 0.4148 (Fig. 2).

\section{Analysis of the association of survival with SYT13 and CEA mRNA levels}

Analyses of the association of OS with SYT13 and CEA levels are presented in Supplemental Fig. 1. OS of patients positive for $S Y T 13$ or $C E A$ was significantly shorter compared with those who were negative for $S Y T 13$ or $C E A$ ( $P=0.0002$ and $P<0.0001$, respectively). Five-year OS was $58.3 \%$ for $S Y T 13$-positive patients and $82.8 \%$ for SYT13-negative patients, while 5-year OS was $49.8 \%$ for $C E A$-positive patients and $80.1 \%$ for $C E A$-negative patients.

Twenty-three (12.3\%) patients experienced peritoneal recurrence, of which 10 had positive peritoneal

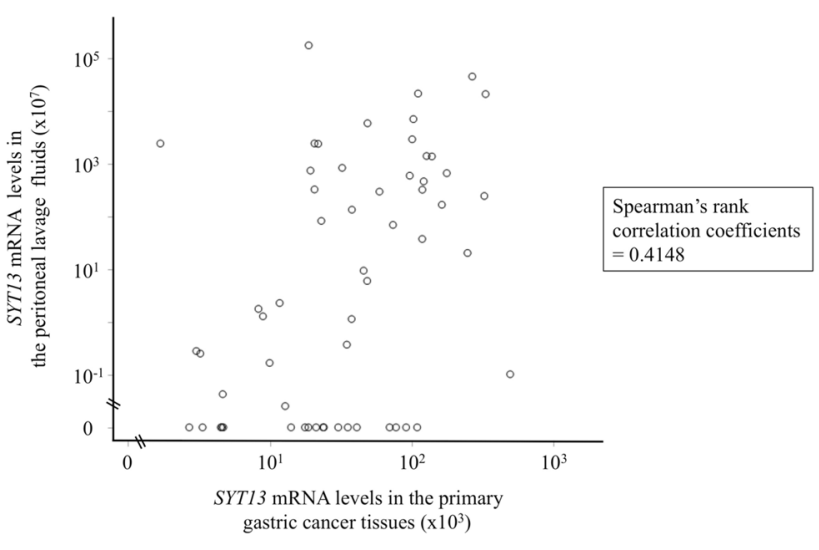

Fig. 2 Correlation of SYT13 mRNA levels between peritoneal lavage fluid and corresponding primary gastric cancer tissues. SYT13 mRNA expression in corresponding primary gastric cancer tissues was obtained from 58 cases. The Spearman's rank correlation coefficient between the levels of SYT13 in peritoneal lavage fluid and primary gastric cancer tissue was 0.4148

lavage cytology. PRFS was significantly shorter for SYT13-positive patients compared with those who were negative $(P<0.0001$, Supplemental Fig. 1a). The 3 -year PRFS values were $69.5 \%$ for $S Y T 13$-positive patients and 95.5\% for SYT13-negative patients. CEA-positive patients experienced shorter PRFS compared with $C E A$-negative patients $(P<0.0001$, Supplemental Fig. 1b). The 3-year PRFS values were $64.8 \%$ for $C E A$-positive and $92.0 \%$ for CEA-negative patients. 
Patients were stratified according to SYT13 and CEA mRNA levels as follows: Ninety-four patients were negative for SYT13 and CEA mRNA (PCR-double negative), and 64 patients were positive for SYT13 or CEA mRNA (PCR-single positive). Twenty-nine patients were positive for $S Y T 13$ and $C E A$ mRNA (PCR-double positive). PRFS was significantly shorter for PCR-double positive, PCR-single positive, and PCR-double negative patients, in descending order $(P<0.0001$, Supplemental Fig. 1c), with 3-year PRFS rates of $97.4 \%, 80.4 \%$, and $52.4 \%$, respectively. The hazard ratios (HRs) for PRFS compared with the PCR-double negative group were 6.63 (95\% confidence interval [CI] 1.93-30.48) and 23.28 (95\% CI 6.85-108.53) in the PCR-single positive and PCR-double positive groups, respectively. Univariate analysis identified significant prognostic factors for PRFS were as follows: tumor depth, lymph node metastasis, macroscopic type, growth type, tumor differentiation, SYT13 positivity, CEA positivity, and CY1. Multivariate analysis identified significant prognostic factors for PRFS as tumor depth, lymph node metastasis, tumor differentiation, and SYT13 positivity (Supplemental Table 3).

(a)

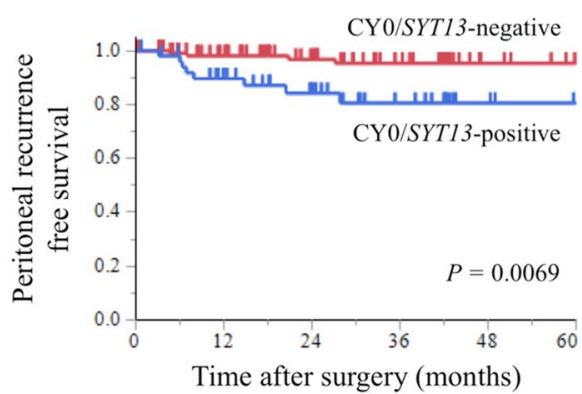

Number at risk $\begin{array}{lllllll}\text { CY0/SYT13-negative } & 111 & 94 & 72 & 57 & 42 & 37\end{array}$ $\begin{array}{lllllll}\text { CY0/SYT13-positive } & 51 & 38 & 27 & 17 & 8 & 5\end{array}$
PRFS of patients with negative peritoneal lavage cytology (CY0).

One-hundred and sixty-two patients were CY0. We next focused on this cohort to evaluate the predictive value of SYT13 and CEA mRNA levels in peritoneal lavage samples after curative gastrectomy (R0 resection). Thirteen (8.2\%) patients experienced peritoneal recurrence after surgery. PRFS was significantly shorter for patients who were SYT13-positive compared with those who were negative $(P=0.0069$, Fig. 3a). The 3 -year PRFS values were $69.5 \%$ for SYT13-positive and $95.6 \%$ for SYT13-negative patients. Patients who were $C E A$ positive experienced significantly shorter PRFS compared with those who were negative $(P=0.021$, Fig. 3b). The 3 -year PRFS rates were $79.3 \%$ for $C E A$-positive and $93.4 \%$ for $C E A$-negative patients.

Patients were stratified according to their levels of SYT13 and CEA mRNA. Ninety-one patients were negative for SYT13 or CEA (CY0/PCR-double negative). Fifty-nine were positive for $S Y T 13$ or $C E A$ (CY0/PCR-single positive). Twelve patients were positive for SYT13 and CEA (CY0/ PCR-double positive). PRFS was significantly shorter for

(c)

(b)

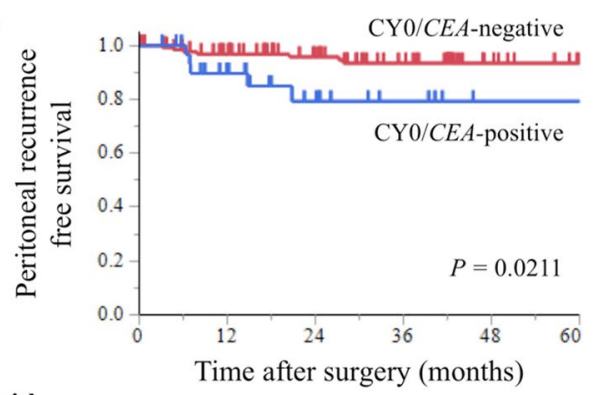

Number at risk

$\begin{array}{lllllll}\text { CY0/CEA-negative } & 130 & 110 & 86 & 68 & 48 & 40\end{array}$

$\begin{array}{lllllll}\text { CY0/CEA-positive } & 32 & 22 & 13 & 6 & 2 & 2\end{array}$
Number at risk

CY0/PCR-double negative

CY0/PCR-single positive

CY0/PCR-double positive

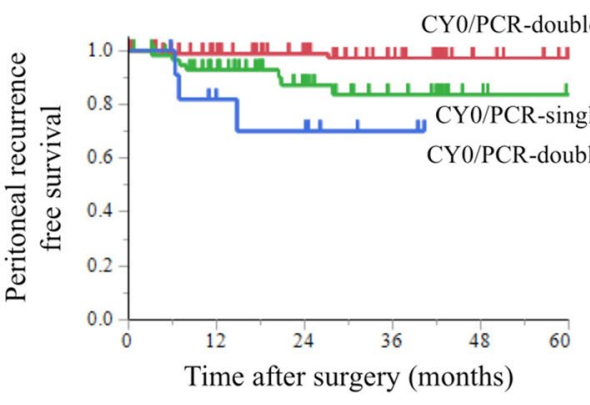

CY0/PCR-double negative

$\begin{array}{cccccc}91 & 79 & 65 & 53 & 40 & 35 \\ 59 & 46 & 28 & 19 & 10 & 7 \\ 12 & 7 & 5 & 2 & 0 & 0\end{array}$

Fig. 3 Analysis of the association of peritoneal recurrence-free survival with SYT13 and CEA mRNA levels in 162 gastric cancer patients with undetectable peritoneal metastasis who were negative for peritoneal lavage cytology (CY0). aSYT13 and bCEA mRNA levels. Double-positive patients had a significantly poorer prognosis compared with negative patients. c Patients were stratified according to their SYT13 and CEA mRNA levels. PRFS was significantly shorter in PCR-double negative, PCR-single positive, and PCR-double positive patients, in descending order 
CY/PCR-double positive, CY0/PCR-single positive, and CY0PCR-double negative patients, in descending order $(P=0.0021$, Fig. 3c). The 3-year PRFS values were as follows: CY0/PCR-double negative, 97.3\%; CY0/PCR-single positive, $83.7 \%$; and CY0/PCR-double positive, $70.1 \%$. The survival curve of the CY0/PCR-double positive group was not significantly different from that of the CY1 group (Supplemental Fig. 3). The HRs for PRFS compared with the CY/PCR-double negative group were 4.76 (95\% CI 1.30-22.49) and 12.27 (95\% CI 2.19-69.77) in the CY0/ PCR-single positive and CY0/PCR-double positive groups, respectively. Univariate analysis identified significant predictive factors for PRFS as follows: larger tumor size, pT4, lymph node metastasis, invasive growth type, tumor differentiation, SYT13 positivity, and CEA positivity. Multivariate analysis identified pT4 and $S Y T 13$ as independent prognostic factors for PRFS (Table 2).

\section{PRFS according to SYT13 and CEA mRNA levels in patients with $\mathrm{CY} 1$}

We focused on 25 patients with CY1. The median followup after surgery was 56.8 months or until death. Among them, ten (40\%) patients experienced peritoneal recurrence. Patients who were $S Y T 13$ positive experienced significantly shorter PRFS compared with those who were SYT13 negative $(P=0.0316$, Fig. 4a), although there was no significant association with $C E A$ levels $(P=0.1606$, Fig. 4b). Peritoneal recurrence was not experienced by all four patients who were SYT13 negative (negative predictive value, 100\%) and by five of seven patients who were CEA negative (negative predictive value, $71.4 \%$ ). These results indicated that SYT13 negativity was a favorable prognostic indicator, even for patients with CY1.

(a)

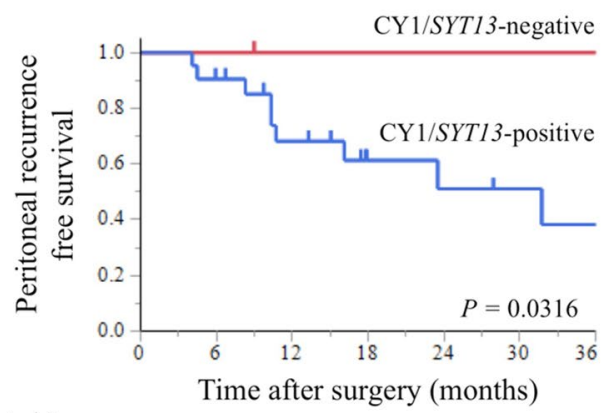

Number at risk

$\begin{array}{llllllll}\text { CY1/SYT13-negative } & 4 & 4 & 3 & 3 & 3 & 3 & 3\end{array}$

$\begin{array}{llllllll}\text { CY1/SYT13-positive } & 21 & 18 & 12 & 6 & 5 & 4 & 3\end{array}$

Fig. 4 Analysis of the association of peritoneal recurrence-free survival with SYT13 and CEA mRNA levels in 25 patients with gastric cancer patients with positive peritoneal lavage cytology (CY1). aSYT13 and bCEA mRNA levels. SYT13-positive patients had signifi-

\section{Subgroup analysis of the association of PRFS with SYT13 and CEA mRNA levels}

We focused on 63 patients with stage III gastric cancer. Among them, 12 (19\%) patients experienced peritoneal recurrence. Although there was no significant difference, PRFS was shorter for patients who were SYT13 positive compared with those who were $S Y T 13$ negative $(P=0.0609$, Supplemental Fig. 4a), and patients who were $C E A$ positive experienced shorter PRFS compared with those who were $C E A$ negative $(P=0.2773$, Supplemental Fig. $4 \mathrm{~b})$.

Next, we evaluated the prognostic effect of postoperative S-1 based chemotherapy (mainly S-1 monotherapy or S-1 plus cisplatin) in 127 patients with stage II/III or P0CY1 without other distant metastases. Among 60 patients without postoperative S-1 based chemotherapy, PRFS was significantly shorter for patients who were $S Y T 13$ positive compared with those who were SYT13 negative $(P=0.0268)$, and patients who were $C E A$ positive experienced shorter PRFS compared with those who were $C E A$ negative $(P=0.1545$, Supplemental Fig. 5a). However, among 67 patients with postoperative S- 1 based chemotherapy, although the difference was marginal, PRFS was shorter for patients who were SYT13 positive $(P=0.0604)$ and there was a significant difference in $C E A$ levels ( $P=0.0023$, Supplemental Fig. 5b).

\section{Discussion}

The development of peritoneal metastasis is a multistep process that commences with gastric cancer cells seeding the peritoneum, surviving in the microenvironment of the abdominal cavity, adhering to the distant mesothelium, invading the basement membrane, and inducing angiogenesis as the tumor grows [16]. The instability of the cancer

(b)

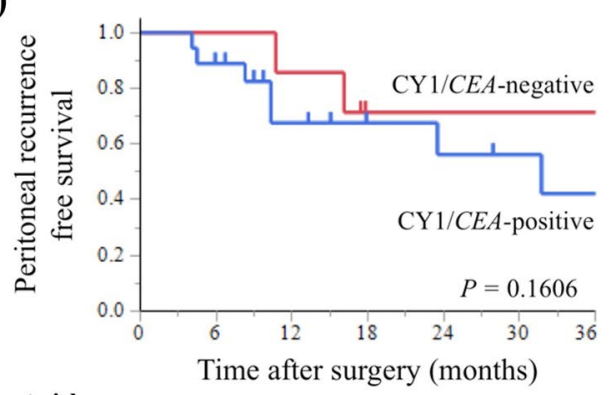

Number at risk

$\begin{array}{llllllll}\text { CY1/CEA-negative } & 7 & 7 & 6 & 3 & 3 & 3 & 3\end{array}$ CY1/CEA-positive $\begin{array}{lllllll}18 & 15 & 9 & 6 & 5 & 4 & 3\end{array}$

cantly poorer prognoses compared with negative patients. There was no significant association with CEA mRNA levels. Peritoneal recurrence was not detected in four of four SYT13-negative patients 
cell genome and the phenotypic heterogeneity of tumor cells contribute to the ability of cancer cells to survive, proliferate, and develop peritoneal metastasis [24]. Once peritoneal seeding occurs, it is associated with poor survival; thus, early detection of free cancer cells in the peritoneum may help prevent peritoneal metastasis and consequently prolong survival.

SYT13 resides on human chromosome $11 \mathrm{p} 11.2$ and encodes a predicted single-pass $47-\mathrm{kDa}$ transmembrane protein $[25,26]$. A previous study reported that $S Y T 13$ expression is high in the brain and gallbladder, and low in the gastric mucosa and peritoneal epithelium [27]. In addition, in this study, we revealed that the expression levels of SYT13 mRNA in the lavage fluid of patients with non-malignant disease (control samples) were low, indicating that its expression in the peritoneal cavity is usually very low or absent. Insubstantial knowledge of SYT13 in oncology was recently enhanced by our findings that SYT13 mRNA levels in gastric cancer tissue reflect the presence of peritoneal micrometastases, and that inhibition of $S Y T 13$ expression in a gastric cancer cell line transfected with an SYT13-specific siRNA (siSYT13) is associated with decreased cell invasion and migration, but not with proliferation and apoptosis. Moreover, they demonstrated that intraperitoneal administration of siSYT13 to mice significantly inhibited the growth of peritoneal nodules and prolonged survival [17]. These findings indicate that SYT13 contributes to the survival of free cancer cells in the peritoneum and to the formation of peritoneal metastasis in patients with gastric cancer. In an attempt to incorporate these findings to the clinic, we were able to reveal a correlation between SYT13 mRNA expression levels in peritoneal lavage fluid and peritoneal recurrence of gastric cancer. Furthermore, we found a positive correlation of SYT13 expression levels in corresponding primary gastric cancer tissue and peritoneal lavage fluid, indicating that expression in lavage fluid represented free cancer cells spilled from the primary gastric cancer tissues. However, taking into consideration the heterogeneity of gastric tumors between the deepest invasive tumor regions and that sampled by biopsy, and the fact that microdissection of samples was not routinely undertaken, the findings may not always be consistent.

Genetic detection using the RT-PCR method has previously been applied to detect micrometastases of gastric cancer [7-9]. Kodera et al. reported that patients with $C E A$ expression in peritoneal lavage fluid had significantly worse overall survival and recurrence-free survival compared with those with negative $C E A$ expression, regardless of lavage cytology results, and concluded that RT-PCR may increase the accuracy of detection of free cancer cells in the peritoneum compared with conventional cytology [7]. Abundant evidence indicates that $C E A$ is the most effective biomarker for analyzing gastric cancer $[8,9]$. Nevertheless, $C E A$ assays are insufficient at low levels because macrophages, leukocytes, and necrotic cancer cells are detected as false positives $[7,28]$. Several studies addressed these weaknesses using high-sensitivity assays that incorporate multiple markers [10-14]. However, there have been no convincing partner markers that can compensate for the weakness of $C E A$. Here, we demonstrate the value of measuring SYT13 mRNA levels in peritoneal lavage fluid for predicting peritoneal recurrence of gastric cancer compared with that of CEA mRNA. In addition to having an individual predictive value for peritoneal recurrence, a combination of the two markers resulted in enhanced performance by the increased specificity.

One strength of our study was its ability to predict peritoneal recurrence even in patients with negative peritoneal lavage cytology. Peritoneal lavage cytology is limited by its relatively low sensitivity, which may fail to detect recurrence in patients with negative lavage cytology. Moreover, we show here that combining the measurements of $C E A$ and SYT13 mRNA had a superior predictive value for peritoneal recurrence compared with each single marker alone. Therefore, our assays may be useful as an eligibility criterion and for allocating factors in clinical trials of aggressive adjuvant chemotherapy designed to prevent and treat peritoneal recurrence (e.g., scirrhous gastric cancer). Another strength of our study is that the measurement of SYT13 mRNA in lavage fluid may increase the options for diagnostic tools. A positive correlation was made between the expression levels in corresponding primary gastric cancer tissue and in lavage fluid samples. While lavage fluid is difficult to collect except during gastrectomy or staging laparoscopy, it can be collected even after gastrectomy.

Determination of the cutoff of molecular markers detected in peritoneal lavage fluid has been recognized as challenging due to dilution of the target cells by normal peritoneal mesothelium cells [7, 15, 28]. Most previous studies suffered from a limited number or absence of control samples from healthy individuals $[7,11,12,15]$. Although we collected 30 benign disease controls and set the cutoff at the highest levels with the aim of minimizing of false positives, much improvement is still required for the optimization of cutoff values.

Moreover, despite the relatively small number of subjects, we acquired convincing evidence that $S Y T 13 \mathrm{mRNA}$ serves as a predictive biomarker for peritoneal recurrence in patients with CY1. Moreover, peritoneal recurrence was not detected in all four SYT13-negative patients examined. Therefore, the high negative predictive value of this assay indicates the diagnostic significance of PCR data for patients with CY1. CY1 is classified as metastatic disease (M1) in the 8th edition of the American Joint Committee on Cancer tumor node metastasis (TNM) staging system for gastric cancer [22] and the Japanese classification of gastric cancer [29]. However, certain patients, despite their CY1 status, achieve long-term survival but do not develop peritoneal 
metastasis. Unfortunately, it is not possible to evaluate the risk of peritoneal recurrence and the status of peritoneal micrometastases in such patients. In the present study, no peritoneal recurrence occurred in four patients with negative SYT13 mRNA (i.e., $100 \%$ of the negative predictive value) and two underwent no postoperative adjuvant treatment. These data either indicated false-positive results of CY or that analysis of $S Y T 13 \mathrm{mRNA}$ can discriminate inviable free cancer cells.

The need for precision medicine has increased in recent years, for example, intraperitoneal chemotherapy (IP) with systemic chemotherapy, where assays with high sensitivity and high specificity are needed to evaluate patients' responses to chemotherapy. A meta-analysis of the effectiveness of this protocol when administered to patients with gastric cancer was previously conducted [30]. Patients with gastric cancer who are treated with IP have a high rate of negative conversion of lavage cytology [31]. Further, even when surgery was reconsidered after conversion of positive cytology to negative, the recurrence of peritoneal metastasis after surgery was frequent [32]. Thus, novel and useful biomarkers that reflect a solid response to IP chemotherapy are needed [32]. Patients who receive IP chemotherapy have an intraabdominal access port, which can allow repeated collection of lavage fluid even after gastrectomy. This may become a new method to evaluate response to treatment if temporal changes in molecular markers in lavage fluid are correlated with therapeutic effects. To address this problem, we have begun to prospectively collect peritoneal lavage fluid to determine if our combination assays can evaluate response to treatment.

This study has several limitations including its relatively small sample size, heterogeneity of postoperative treatment, and retrospective design. Sample collection was limited at gastrectomy. Further, the statistical power of the study might be insufficient with respect to the analysis of subgroups with positive lavage cytology. A prospective large-scale observational study and external validation will, therefore, be required to determine whether the assay can be translated to the clinic. Finally, qRT-PCR assays are time-consuming (2-3 $\mathrm{h}$ for each run and more for combined markers). Thus, a multiplex assay may prove to be more clinically useful.

\section{Conclusions}

SYT13 and CEA mRNA levels, alone or in combination, in peritoneal lavage fluid are promising biomarkers for predicting peritoneal recurrence of gastric cancer, even in patients who undergo curative gastrectomy.

Acknowledgements We thank Edanz Group (www.edanzediting.com/ ac) for editing a draft of this manuscript.

\section{Compliance with ethical standards}

Conflict of interest The authors declare that they have no competing interests.

Ethical approval All procedures performed were in accordance with the ethical standards of the responsible committee on human experimentation (institutional and national) and with the Helsinki Declaration of 1964 and later versions.

Informed consent Informed consent was obtained from all individual participants included in the study.

\section{References}

1. Ferlay J, Soerjomataram I, Dikshit R, Eser S, Mathers C, Rebelo $\mathrm{M}$, et al. Cancer incidence and mortality worldwide: sources, methods and major patterns in GLOBOCAN 2012. Int J Cancer. 2015;136:E359-E386386.

2. Shen L, Shan YS, Hu HM, Price TJ, Sirohi B, Yeh KH, et al. Management of gastric cancer in Asia: resource-stratified guidelines. Lancet Oncol. 2013;14:e535-e547547.

3. Van Cutsem E, Sagaert X, Topal B, Haustermans K, Prenen H. Gastric cancer. Lancet (London, England). 2016;388:2654-64.

4. Kodera Y, Yamamura Y, Shimizu Y, Torii A, Hirai T, Yasui K, et al. Peritoneal washing cytology: prognostic value of positive findings in patients with gastric carcinoma undergoing a potentially curative resection. J Surg Oncol. 1999;72:60-4; discussion 4-5.

5. Bando E, Yonemura Y, Takeshita Y, Taniguchi K, Yasui T, Yoshimitsu Y, et al. Intraoperative lavage for cytological examination in 1,297 patients with gastric carcinoma. Am J Surg. 1999;178:256-62.

6. Sasako M, Sakuramoto S, Katai H, Kinoshita T, Furukawa $\mathrm{H}$, Yamaguchi T, et al. Five-year outcomes of a randomized phase III trial comparing adjuvant chemotherapy with S-1 versus surgery alone in stage II or III gastric cancer. J Clin Oncol. 2011;29:4387-93.

7. Kodera Y, Nakanishi H, Ito S, Yamamura Y, Kanemitsu Y, Shimizu Y, et al. Quantitative detection of disseminated free cancer cells in peritoneal washes with real-time reverse transcriptasepolymerase chain reaction: a sensitive predictor of outcome for patients with gastric carcinoma. Ann Surg. 2002;235:499-506.

8. Deng K, Zhu H, Chen M, Wu J, Hu R, Tang C. Prognostic significance of molecular analysis of peritoneal fluid for patients with gastric cancer: a meta-analysis. PLoS ONE. 2016;11:e0151608.

9. Virgilio E, Giarnieri E, Giovagnoli MR, Montagnini M, Proietti A, D'Urso R, et al. Gastric cancer cells in peritoneal lavage fluid: a systematic review comparing cytological with molecular detection for diagnosis of peritoneal metastases and prediction of peritoneal recurrences. Anticancer Res. 2018;38:1255-62.

10. Jeon $\mathrm{CH}$, Kim IH, Chae HD. Prognostic value of genetic detection using CEA and MAGE in peritoneal washes with gastric carcinoma after curative resection: result of a 3-year follow-up. Medicine (Baltimore). 2014;93:e83.

11. Li Z, Zhang D, Zhang H, Miao Z, Tang Y, Sun G, et al. Prediction of peritoneal recurrence by the mRNA level of CEA and MMP-7 in peritoneal lavage of gastric cancer patients. Tumour Biol. 2014;35:3463-70.

12. Kodera Y, Nakanishi H, Ito S, Yamamura Y, Fujiwara M, Koike $\mathrm{M}$, et al. Prognostic significance of intraperitoneal cancer cells in gastric carcinoma: detection of cytokeratin 20 mRNA in 
peritoneal washes, in addition to detection of carcinoembryonic antigen. Gastric Cancer. 2005;8:142-8.

13. Tamura N, Iinuma H, Takada T. Prospective study of the quantitative carcinoembryonic antigen and cytokeratin 20 mRNA detection in peritoneal washes to predict peritoneal recurrence in gastric carcinoma patients. Oncol Rep. 2007;17:667-72.

14. Mori K, Suzuki T, Uozaki H, Nakanishi H, Ueda T, Matsuno $\mathrm{Y}$, et al. Detection of minimal gastric cancer cells in peritoneal washings by focused microarray analysis with multiple markers: clinical implications. Ann Surg Oncol. 2007;14:1694-702.

15. Katsuragi K, Yashiro M, Sawada T, Osaka H, Ohira M, Hirakawa K. Prognostic impact of PCR-based identification of isolated tumour cells in the peritoneal lavage fluid of gastric cancer patients who underwent a curative $\mathrm{R} 0$ resection. Br J Cancer. 2007;97:550-6.

16. Kanda M, Kodera Y. Molecular mechanisms of peritoneal dissemination in gastric cancer. World J Gastroenterol. 2016;22:6829-40.

17. Kanda M, Shimizu D, Tanaka H, Tanaka C, Kobayashi D, Hayashi $\mathrm{M}$, et al. Synaptotagmin XIII expression and peritoneal metastasis in gastric cancer. Br J Surg. 2018;105:1349-58.

18. Kanda M, Shimizu D, Fujii T, Sueoka S, Tanaka Y, Ezaka K, et al. Function and diagnostic value of Anosmin-1 in gastric cancer progression. Int J Cancer. 2016;138:721-30.

19. Kanda M, Nomoto S, Oya H, Shimizu D, Takami H, Hibino S, et al. Dihydropyrimidinase-like 3 facilitates malignant behavior of gastric cancer. J Exp Clin Cancer Res. 2014;33:66.

20. Kanda M, Shimizu D, Tanaka H, Tanaka C, Kobayashi D, Hayashi M, et al. Significance of SYT8 For the Detection, Prediction, and treatment of peritoneal metastasis from gastric cancer. Ann Surg. 2018;267:495-503.

21. Umeda S, Kanda M, Miwa T, Tanaka H, Tanaka C, Kobayashi D, et al. Expression of sushi domain containing two reflects the malignant potential of gastric cancer. Cancer Med. 2018;7:5194-204.

22. Liu JY, Peng CW, Yang XJ, Huang CQ, Li Y. The prognosis role of AJCC/UICC 8(th) edition staging system in gastric cancer, a retrospective analysis. Am J Trans Res. 2018;10:292-303.

23. Japanese Gastric Cancer A. Japanese gastric cancer treatment guidelines 2014 (ver. 4). Gastric Cancer. 2017;20:1-19.
24. Janjigian YY, Kelsen DP. Genomic dysregulation in gastric tumors. J Surg Oncol. 2013;107:237-42.

25. von Poser C, Sudhof TC. Synaptotagmin 13: structure and expression of a novel synaptotagmin. Eur J Cell Biol. 2001;80:41-7.

26. Fukuda M, Mikoshiba K. Characterization of KIAA1427 protein as an atypical synaptotagmin (Syt XIII). Biochem J. 2001;354:249-57.

27. Fagerberg L, Hallstrom BM, Oksvold P, Kampf C, Djureinovic $\mathrm{D}$, Odeberg $\mathrm{J}$, et al. Analysis of the human tissue-specific expression by genome-wide integration of transcriptomics and antibodybased proteomics. Mol Cell Proteom. 2014;13:397-406.

28. Nakanishi H, Kodera Y, Yamamura Y, Ito S, Kato T, Ezaki T, et al. Rapid quantitative detection of carcinoembryonic antigenexpressing free tumor cells in the peritoneal cavity of gastriccancer patients with real-time RT-PCR on the lightcycler. Int J Cancer. 2000;89:411-7.

29. Japanese Gastric Cancer A. Japanese classification of gastric carcinoma: 3rd English edition. Gastric Cancer. 2011;14:101-12.

30. Yang S, Feng R, Pan ZC, Jiang T, Xu Q, Chen Q. A Comparison of intravenous plus intraperitoneal chemotherapy with intravenous chemotherapy alone for the treatment of gastric cancer: a metaanalysis. Sci Rep. 2015;5:12538.

31. Ishigami H, Fujiwara Y, Fukushima R, Nashimoto A, Yabusaki $\mathrm{H}$, Imano $\mathrm{M}$, et al. Phase III Trial comparing intraperitoneal and intravenous paclitaxel plus S-1 Versus cisplatin plus S-1 in patients with gastric cancer with peritoneal metastasis: PHOENIX-GC trial. J Clin Oncol. 2018;36:1922-9.

32. Yamaguchi H, Kitayama J, Ishigami H, Kazama S, Nozawa H, Kawai K, et al. Breakthrough therapy for peritoneal carcinomatosis of gastric cancer: intraperitoneal chemotherapy with taxanes. World J Gastroint Oncol. 2015;7:285-91.

Publisher's Note Springer Nature remains neutral with regard to jurisdictional claims in published maps and institutional affiliations. 